\title{
Évitement et médiation rituelle à Wallis (Polynésie occidentale)
}

\section{Sophie Chave-Dartoen}

\section{OpenEdition}

1 Journals

\section{Édition électronique}

URL : https://journals.openedition.org/clo/1259

DOI : $10.4000 /$ clo. 1259

ISSN : 2266-1816

Éditeur

INALCO

\section{Édition imprimée}

Date de publication : 30 décembre 2011

Pagination : 37-64

ISBN : 978-2-85831-202-3

ISSN : 0396-891X

\section{Référence électronique}

Sophie Chave-Dartoen, «Évitement et médiation rituelle à Wallis (Polynésie occidentale) », Cahiers de littérature orale [En ligne], 70 | 2011, mis en ligne le 17 mars 2013, consulté le 01 juillet 2021. URL: http://journals.openedition.org/clo/1259; DOl : https://doi.org/10.4000/clo.1259

Ce document a été généré automatiquement le 1 juillet 2021.

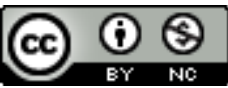

Cahiers de littérature orale est mis à disposition selon les termes de la Licence Creative Commons Attribution - Pas d'Utilisation Commerciale 4.0 International. 


\title{
Évitement et médiation rituelle à Wallis (Polynésie occidentale)
}

\author{
Sophie Chave-Dartoen
}

\section{Expression des relations asymétriques}

1 Certaines relations demandent, en Océanie, des formes spécifiques d'expression et de médiation (Brenneis et Myers, 1989). La parole prend ici un caractère particulier, exigeant la maitrise des formulations convenables et, bien souvent, la ressource d'un répertoire étendu d'images, de métaphores, d'expressions permettant d'exprimer de façon détournée des idées, des suggestions, des prières que l'on ne pourrait mentionner de façon directe, en des circonstances non cadrées par un protocole rigoureux. La parole est généralement, dans de tels contextes, doublement indirecte : tropes, figures de style, mais aussi configuration relationnelle de la situation d'interlocution (cf. ce que Goffman a défini comme un "cadre de l'expérience ») donnent à la parole, dans cet écart entre le dit et le signifié, une efficacité singulière. En outre, dans la plupart des cas, cette parole ne saurait être directement et immédiatement adressée à son destinataire : un médiateur est nécessaire qui, maîtrisant les formes du discours, en maîtrise aussi l'usage et la portée, comme je me propose de le montrer ici sur la base d'un exemple wallisien'.

2 L'ethnographie de la région fait état d'institutions spécifiquement destinées à la maîtrise du rituel, et plus particulièrement de la parole dans la manifestation et l'expression des relations particulières. Les orateurs cérémoniels forment, dans les sociétés kanak de Nouvelle-Calédonie (Monnerie, 2005) un exemple de cette parole, mesurée, codifiée et particulièrement efficace qui ne saurait être prononcée autrement que par l'intermédiaire de spécialistes reconnus et respectés de tous. C'est cependant en Polynésie occidentale que ce type de phénomène est à la fois très net et bien documenté. L'attention sera portée ici sur l'importance structurelle de l'institution que constituent ces officiants, le contraste se dessinant clairement avec l'absence d'une institution comparable en Polynésie orientale où, en revanche, une classe apparaissant 
comme plus nettement cléricale s'est développée (O’Reilly, 1948; Sahlins, 1995 pour le cas hawaïen).

3 Les travaux d'Alessandro Duranti $(1988,1990,1994)$ analysent de façon très fine les interactions verbales au sein des conseils de village à Samoa ${ }^{2}$. Il a pu approfondir la question du rôle singulier des "chefs» de lignage matai, et de ceux qui, prenant la parole en leur nom, portent des titres leur conférant les fonctions d'orateurs cérémoniels, tuafale (1990). Il a ainsi mis l'accent sur la dimension éminemment politique de cette fonction et de la parole qui, en conseil de village, est performative, c'est-à-dire proprement instituante, car collectivement et formellement validée.

4 À Tonga, il existait autrefois auprès de chaque « chef " ('eiki) des assistants cérémoniels dont le rôle essentiel était la direction de la cérémonie du kava, mais dont les responsabilités s'étendaient à la transmission des récits, la surveillance et la protection, le conseil, la modération, la dénonciation des mésactions auprès des chefs ${ }^{3}$. Ces hommes, issus des lignages aînés ou d'origine étrangère, étaient libres des « interdits » (tapu) liés à la personne des chefs, et sont présentés par les traditions comme potentiellement conspirateurs et assassins. Cette configuration particulière rapproche officiants tongiens et orateurs samoans en ce que chacun forme, avec le chef qu'il accompagne, une entité constituée des deux faces du pouvoir: l'action par la force, efficace par la domination qu'elle instaure, mais ouverte sur l'arbitraire pour le chef; l'action par la parole, efficace par son caractère instituant, dangereuse si elle n'est pas correctement maîtrisée, pour son officiant.

5 La fonction de tels assistants cérémoniels est attestée à Wallis bien qu'ayant une moindre importance dans l'organisation des relations sociales en général, et notamment dans l'organisation de la chefferie. Il est probable, comme nous le verrons, que l'influence tongienne sur cette institution explique la similitude relative des fonctions et des dénominations de ces officiants. Le cas wallisien permettra toutefois d'approfondir l'étude d'un aspect de ces chefferies polynésiennes, les assistants cérémoniels manifestant, outre la dualité du pouvoir et la complémentarité de ses deux aspects (régulation par la force/ régulation par la parole), la nécessité d'une médiation permettant une relation bénéfique entre vivants et ancêtres/déités. Dans une telle configuration, analysée par Hocart (1978) dans le cadre de son étude des royautés sacrées, les uns et les autres relèvent, dans la complémentarité et l'interdépendance, de l'organisation et de l'ordre du cosmos propre à cette région du Pacifique. Cette médiation, en instaurant momentanément la conjonction contrôlée de ces deux ordres imbriqués, mais distincts, organise une distance qui interdit toute relation directe et immédiate. Elle indique ainsi le pôle supérieur de la relation momentanément formalisée. En m'intéressant aux conditions de la parole indirecte, je compte explorer ici, avec l'ordre du cosmos wallisien, la fonction du chef suprême (hau) commodément dénommé « roi ${ }^{4}$ ». Différentes sources bibliographiques et des données rassemblées au cours de divers terrains menés à Wallis depuis 1990 sont mobilisées, mais cet article repose avant tout sur un long entretien avec Nikola Vaitanoa (juillet 2010) dont toutes les citations sont extraites. La fonction d'assistant cérémoniel (molofaha) que Nikola Vaitanoa assure auprès du roi de Wallis depuis plus de vingt ans fait de lui le meilleur spécialiste de la question et, comme nous allons le voir, une autorité en la matière. Une large place est laissée à ses explications et à son expérience tout à fait exceptionnelle. 


\section{L'expression du respect et l'évitement dans les relations asymétriques}

6 À Wallis, toute occasion formelle (visites et départs, réunions, festivités religieuses...) implique un contrôle de l'espace, de la parole ainsi que diverses formes d'évitement et de médiation. Ces occasions, en effet, révèlent la distinction en responsabilités et en statut des personnes et des groupes, rendant perceptible à l'assistance leur complexe hiérarchie, toujours questionnée, toujours travaillée.

7 L'évitement se dit faka'apa'apa. Ce terme signifie aussi « respect » en ce que la distance spatiale, le maintien d'une position abaissée, l'abstention de contact physique et visuel, manifestent la reconnaissance d'une supériorité de statut (Firth, 1970, concernant Tikopia). Il doit en aller ainsi en toute occasion où l'on se trouve en présence de supérieurs : pour l'enfant face à l'adulte, le cadet face à l'aîné, un homme face à sa sœur, un roturier face à un aristocrate, un vivant face aux morts... L'asymétrie du statut est nette et clairement signifiée. Il en va de même, pour tous, face à Dieu dans le culte catholique local, et face au roi, chef suprême dans la hiérarchie coutumière wallisienne.

Cette reconnaissance, qu'accentuent l'usage d'un langage approprié, d'un ton mesuré, et la préséance dans les tours de parole ${ }^{5}$, n'est cependant pas seulement formelle. La distance spatiale assure une forme de protection réciproque, mais non symétrique entre entités de statuts, et donc de potentialités différentes (Chave-Dartoen, 1997). En désignant le pôle supérieur en statut, les marques de respect signalent celui des deux pôles en relation la plus directe aux ancêtres et à Dieu. Ce pôle est ainsi reconnu comme dépositaire de l'autorité des ancêtres et de la puissance divine dont dépendent, avec lui, ses inférieurs. En position d'intermédiaire, il contrôle les conditions de leur survie, il assure leur bien-être et peut leur causer des infortunes. La réciproque de cette relation est donc l'évitement respectueux, par gratitude et/ou par crainte de sanction, mais aussi et surtout afin d'assurer la préservation des caractères bénéfiques d'une relation avec un être supérieur dont la position intermédiaire et le caractère "séparé » $\left(\operatorname{tapu}^{6}\right)$ doivent être préservés afin de rester efficaces.

9 La distance spatiale, l'usage d'un registre particulier du langage et le réglage des tours de parole sont, dans certains cas, complétés par la nécessaire médiation d'officiants cérémoniels. C'est le cas des messes catholiques, où les prêtres font office de médiateurs entre les fidèles et Dieu. C'est également le cas des cérémonies du kava organisées en présence d'une personne titrée (roi ou «chef coutumier» détenteur d'une charge). Ces cérémonies, au cours desquelles on entrait autrefois en contact avec les déités (Bataillon, 1841; Chave-Dartoen, 1992, 2000), sont organisées en deux parties : 1) la préparation - au cours de laquelle un morceau de racine pilé du piper methysticum est malaxé à de l'eau - est accompagnée de formules et de discours qui s'enchaînent selon un protocole précis ; 2) la distribution de la boisson obtenue est faite sous forme de coupes réparties dans l'assistance selon un ordre réglé sur la hiérarchie des fonctions et des statuts. Dans ces occasions, interactions verbales et allocutions atteignent un haut degré de formalisme. Le porteur du titre le plus élevé, à l'intention de qui le kava est préparé, est alors doté d'un assistant (molofaha), qui règle les discours et dirige la cérémonie du kava, mais dont la fonction explicite consiste à le «servir " (tauhi) et à le « protéger» des attaques (taupau). 
À Wallis, si [tu es un visiteur de haut statut et que] tu vas dans un village, il y a toujours le molofaha du village. Il ne se peut pas que tu te rendes à une réunion dans un village où t'attendent le chef et ses villageois sans qu'il y ait de molofaha.

10 Généralement les villages ont leur molofaha appointé, mais si ce n'est pas le cas, on désigne quelqu'un :

Viens là, s'il te plaît... et assieds-toi à côté du chef de village parce que les visiteurs arrivent et qu'il faut les accueillir!

11 Cette règle s'applique à tous les chefs coutumiers en fonction ${ }^{7}$. Il en va cependant un peu différemment du roi, qui porte le nom-titre de Lavelua. Il peut, s’il le désire, arriver avec son propre molofaha:

Si Lavelua me dit [à moi, son molofaha attitré] qu'il se déplace, il peut me dire d'y aller, que nous partions ensemble. Mais s'il va dans un rassemblement où l'on prépare un kava, je suis dans l'obligation d'y aller, indépendamment de son invitation, car c'est mon travail! Pour le reste... si je n'y vais pas, cela [le choix du molofaha] dépend des gens [qui reçoivent le roi].

12 Pour ce qui est de la nécessité d'une assistance cérémonielle dans les occasions formelles, le roi apparaît ainsi comme un cas doublement particulier au regard des autres membres de la chefferie : il dispose, tout d'abord, d'un officiant molofaha attitré dont il peut demander l'escorte; ensuite, aucun kava offert au roi ne peut se faire en l'absence de cet officiant qui est, comme nous allons le voir, garant du bon déroulement de la cérémonie. La raison de cette singularité réside dans la position unique de la figure royale et dans le statut spécifique au porteur du titre suprême. L'examen des responsabilités propres à son officiant cérémoniel permet d'en comprendre certains aspects importants. Il s'agit d'abord de contrôler toute parole formelle adressée au roi, la médiation de l'officiant autorisant, seule, une adresse directe; il s'agit ensuite de diriger la cérémonie du kava royal et d'en verrouiller les moments essentiels ; enfin, il s'agit de demander, pour tous, les bienfaits dont le roi garantit la redistribution générale.

\section{Les fonctions de mu'a et de molofaha : médiation cérémonielle et protection de la position royale}

13 À Tonga, chaque grand aristocrate détenteur d'un titre avait autrefois auprès de lui son officiant matāpule. Ce dernier, détenteur d'un titre et d'une charge héréditaires, échappait, par son origine (aristocratique et/ou étrangère) aux interdits autrefois relatifs à la personne d'un chef de haut rang (Gifford, 1929, 140). Seul à pouvoir l'approcher et parler à voix haute en sa présence, il distribuait le kava, assurait l'investiture et officiait comme porte-parole (idem, 63-71; Martin, 1981, 291). Martin indique (idem, 204) qu'il faisait office d'intercesseur avec l'au-delà en l'absence de prêtres. Les matāpule constituaient ainsi à Tonga, avec leurs propres dépendants appelés mu'a (descendants, cadets, collatéraux) auxquels ils déléguaient des tâches secondaires, une catégorie particulière d'aristocrates dotés de fonctions rituelles précises auprès du dirigeant de la communauté. Le terme matāpule signifie littéralement, en tongien comme en wallisien, "face-de-chef», ce que signifie également de façon moins explicite le terme $m u^{\prime} a:$ : devant, avant $»^{8}$.

14 Il en va sensiblement de même à Wallis. Tout comme les membres de la chefferie, l'officiant cérémoniel du roi porte normalement un nom-titre transmis dans son 
groupe de descendance, avec la charge. On considère communément à Wallis que la chefferie actuelle ${ }^{9}$ et certaines institutions telles que la cérémonie du kava royal trouvent leur origine à Tonga. Il en va de même concernant la fonction d'officiant cérémoniel :

Ils avaient leurs noms-titres... car [le nom-titre] Teleha'uli est venu de là. À cette époque le distributeur [des chefs arrivés] de Tonga était Teleha'uli. Et c'est resté [le nom-titre et la fonction] dans sa descendance. C'était comme ça à Wallis !

Cependant, Nikola Vaitanoa n'a pas été choisi dans cette descendance, car les règles de succession se sont assouplies :

Mon nom [Molofaha], il a été choisi en référence à ma fonction de molofaha parce que nous n'appartenons pas à une descendance de distributeurs. Il y a [comme noms-titres et comme descendances où les porteurs sont respectivement choisis] Teleha'uli, il y a Tu'iha'atala et Faikimu'a... Le [nom-titre] Faikimu'a, c'est de là que vient la vaste descendance à laquelle appartenait Fusi Ieti. Cet homme distribuait le kava... il portait le titre de Faikimu'a, le prédécesseur de Tino. Mais mon nom, c'est juste le nom désignant celui qui s'assied près d'un chef pour le protéger.

16 À cette fonction correspond aussi le terme de $m u$ 'a, terme signifiant «avant, devant », et que l'on trouve à Wallis comme à Tonga ${ }^{10}$ :

C'est ainsi à Wallis! On dit de moi que je suis le mu'a de Lavelua. L'homme qui sert [et garde] toujours Lavelua, c'est le mu'a de Lavelua. [...] La raison de cette chose, lorsque le roi voyage, c'est là que se trouve l'origine du nom qui est «mu'a de Lavelua ", lorsque le roi allait quelque part autrefois, il n'y avait pas de voitures, ils allaient [lui et sa suite] à pied. Alors le roi marchait, et l'homme qui avait la même fonction que moi [littéralement «l'homme comme moi »] allait devant. Si quelque chose survenait, il l'arrêtait et demandait aux gens de s'asseoir à terre. Et si l'on devait offrir en route quelque chose au roi pour son bien-être comme une noix de coco, et bien cet homme l'inspectait d'abord! Cet homme vérifiait d'abord, puis cela fait, il le faisait porter au roi.

Plus loin dans l'entretien, N. Vaitanoa précisa qu'il lui revenait, lors des cérémonies, de recevoir les présents offerts au roi (colliers de fleurs, nattes, mais aussi l'annonce des danses...) avant qu'il n'autorise qu'on les lui fasse parvenir.

Contrairement à Tonga où il semble que les mu'a avaient autrefois comme tâche principale d'assurer les distributions sous la direction d'un matāpule de rang supérieur (Martin, 1981: 291; Gifford, 1929, 109), les fonctions de molofaha et de mu'a ne se superposent donc pas exactement à Wallis, bien qu'actuellement un même homme les assume conjointement. Nous allons voir en quoi consistent ses attributions et ses responsabilités avant de revenir à l'examen des relations ainsi organisées et manifestées.

\section{La parole indirecte, ou la maîtrise de la parole adressée au roi}

19 L'accès au roi est "fermé »11 (puipui). Il nécessite des procédures formelles, dont un rendez-vous pris auprès de ses proches, et la présence d'un molofaha. Nikola Vaitanoa n'est pas seul à pouvoir assumer cette fonction. Le défunt roi l'appelait pour toutes les affaires importantes, mais il est arrivé que des chefs coutumiers, porteurs de titre, le remplacent lors des visites et des accueils formels ${ }^{12}$. Les visiteurs arrivent munis d'un présent ma'ukava (un morceau de racine de kava, une bouteille d'alcool et/ou des billets de banque dans une enveloppe) dont on dit qu'il «porte», qu'il «accompagne» la 
parole. Devant le roi comme en toute occasion formelle, cette parole prend la forme d'un discours : après les salutations d'usage, l'information est délivrée en détail. Une formule indique la fin de l'allocution. Il n'est alors plus possible de s'adresser de nouveau au roi à moins d'y être, de nouveau, expressément autorisé.

La discussion avec Nikola Vaitanoa avait débuté sur l'exemple de l'orateur samoan et des formes de médiation qu'il assure, prenant la parole pour un chef ne désirant pas s'exposer à la critique :

À Wallis, le chef parle. Mais quelqu'un vient pour le protéger, il le protège lorsque on [vient]..., lors d'une annonce [par exemple]... pour qu'il autorise... la possibilité de faire une annonce à destination du chef, qu'il permette qu'on lui adresse la parole.

21 Revenant sur ses attributions en tant que molofaha : «Il dépend de moi que l'on fasse, ou que l'on ne fasse pas, de discours. » «Il n'est pas possible, explique-t-il ailleurs, que l'on dise directement au Lavelua : Autorisez-moi, Lavelua, à vous adresser la parole ! » Il ne s'agit pas là d'une simple affaire de convenances. Un bon molofaha est indispensable en ce qu'il prévient les affronts :

Quelqu'un arrive, qui veut parler devant [au roi], alors il me demande que je l'autorise, si je le veux bien, à faire un petit discours. Cela dépend de l'opportunité. Si je veux qu'il fasse un discours, c'est libre [...] il fait son discours. Mais s'il y a un empêchement, alors il ne parle pas. Et si c'est un discours que je sais offensant pour le roi, pour les chefs, alors je dois l'empêcher. C'est moi qui fais cesser, il ne fait pas de discours ce jour-là.

On voit ici très distinctement en quoi le molofaha assure la protection du roi ou du chef auprès de qui il officie. Cette protection consiste à verrouiller toute parole néfaste, soit que le moment soit inapproprié, soit que le molofaha prévienne les intentions malveillantes ou belliqueuses des visiteurs.

Ce contrôle présente toutefois d'autres aspects. En développant l'exemple d'un chef de village (Tu'i Matā'utu) venant apprendre au roi le décès d'un villageois, Nikola Vaitanoa en a montré la complexité : une fois que l'autorisation du molofaha est obtenue :

... le discours que tu formules afin de saluer Lavelua, tu le délivres directement à Lavelua! Ainsi que le message [...] :

- (1) Molofaha, je te prie de m'accorder l'autorisation de rencontrer le seigneur.

Après cela, il présente ses salutations, puis je lui dis :

- (2) Écoutons la nouvelle du [présent] ma’ukava, que le seigneur en prenne connaissance.

Quand j'ai fini, disons, Tu'iMatautu parle et me répond :

- (3) Molofaha, il faut que je fasse savoir au seigneur Lavelua. [Puis s'adressant au roi] (4) « Lavelua voici vos humbles enfants qui accourent vers vous pour apprendre à votre seigneurie... ». Il s'adresse directement au roi, et non plus à moi.

Notons que cette situation, considérée comme exemplaire par mon interlocuteur, ne présente pas le danger potentiel d'une visite moins convenue (tout décès doit être annoncé au roi avant que la nouvelle ne se répande pour la préparation des funérailles). La demande d'autorisation (1) ne rencontre donc pas d'obstacle. Toutefois, l'accord du molofaha (2) est suivi d'une formule (3) mettant en valeur sa médiation : quand bien même la permission est accordée, une dernière adresse est indirecte (3) « Molofaha... ») avant de se tourner vers le roi (4). Apparait bien là une forme de "protection » qui ne prévient pas tant des paroles agressives ou inappropriées qu'une proximité trop grande dans les séquences et dans les registres du discours. La médiation du molofaha assure ici les conditions d'une adresse directe, mais distanciée selon deux modalités: i) la 
transition que sa médiation nécessaire assure (3), et ii) les formules d'adresse au roi qui effacent le sujet parlant derrière un collectif dont l'infériorité statutaire et la dépendance, sont clairement signifiées ${ }^{13}$. Cette médiation, on le voit bien, perd ici une dimension qui pouvait sembler essentiellement "politique» et une dimension davantage rituelle apparait, que confirment deux autres aspects perceptibles dans la forme que prend l'entrevue suite à ce premier échange : le roi peut répondre, par une question ou par un commentaire.

Dans le cas d'un commentaire, l'assistance ponctue chacune de ses phrases par l'exclamation d'une courte locution («koe!») qui signifie «oui » dans le registre de langage relevé ${ }^{14}$. Il s'agit donc, pour les personnes présentes, de marquer un accord sur tout ce que le roi énonce. Dans ce cas, son avis est définitif et peut équivaloir un ordre. En général, la visite se termine là. $\mathrm{Si}$, exceptionnellement, « ils [les visiteurs] souhaitent ajouter quelque chose [une autre information], ils me demandent une nouvelle autorisation", explique Nikola Vaitanoa : «Il y a encore une chose, Molofaha, pour laquelle nous voulons encore te demander l'autorisation de parler. " L'officiant juge, cette fois encore, de l'opportunité de prolonger l'entrevue.

Cependant, si le roi répond par une question, la dimension plus proprement rituelle de la médiation apparaît très nettement dans la modalité de la réponse telle qu'elle doit être formulée. Le roi

[...] leur pose directement la question, et pour lui répondre, ils me demandent à nouveau l'autorisation de faire savoir leur réponse au [roi]... La raison est que nous tous [Wallisiens] nous devons passer par l'intermédiaire de celui qui assiste [et protège] le roi.

Le roi s'adresse directement aux visiteurs s'il parle, mais pour la réponse, une médiation est de nouveau nécessaire : «Molofaha, nous te prions de bien vouloir nous autoriser à faire une petite... [réponse]. Quand j'ai fini, ils font une réponse à la question posée par le roi. » Ici encore donc, il s'agit moins de contrôler le contenu du message que de régler la distance entre les protagonistes: or, cette distance, inexistante lorsque le roi s'adresse à ses visiteurs, s'accrô̂t lorsqu'il s'agit de lui répondre. En ces rares occasions d'entrevues formelles, le recours différencié au molofaha manifeste très clairement l'asymétrie de la relation existant entre le roi et ses visiteurs.

Cette présentation de la fonction du molofaha lors de telles délégations permet de mettre en évidence différentes caractéristiques de la fonction royale et de la parole dans un contexte cérémoniel. Si l'accueil et l'organisation de la parole semblent, en première analyse, constituer un moyen de contrôle politique, une attention plus fine aux interactions permet d'aller plus loin. Elle montre bien, en effet, que le rôle de l'officiant cérémoniel et les ressorts de sa médiation sont avant tout rituel en ce que, si cette médiation protège la fonction royale des graves atteintes qu'elle peut anticiper, cette dernière assure avant tout une mise à distance réglée dans l'échange langagier entre les visiteurs et le chef suprême. Elle signifie, par ce moyen, l'écart de statut, mais assure également la distance nécessaire à la protection des uns et de l'autre contre une promiscuité qui, rompant l'asymétrie de la relation, serait néfaste pour tous.

La valeur performative du discours apparaît, dans ce contexte, selon deux modalités différentes. Tout d'abord, la nécessité d'une médiation en ces circonstances formelles montre que dans de telles occasions, la parole porte davantage que de façon courante et qu'il y a lieu de particulièrement la réguler. Duranti (1988, notamment p. 22 sqq) a bien 
analysé ce phénomène lors des conseils samoans : d'une façon générale, toute parole formulée en ce genre de circonstances publiques engage son énonciateur et opère une action influant sur l'état des relations sociales et sur l'ordre du monde. Il suffit donc, à Wallis, que des personnes en position de destituer le roi (soit par leur statut, soit par le rapport de force) accèdent à de telles occasions et obtiennent l'autorisation de l'officiant cérémoniel, pour qu'elles lui signifient son congé et annoncent son remplacement ${ }^{15}$. Ainsi peut-on comprendre l'organisation des salutations formelles qui, énumérant les présents par ordre de préséance, marque le respect, mais surtout entérine leur présence et leur reconnaît le droit de participer pleinement à l'événement en prenant la parole le moment requis ${ }^{16}$. Il y a ensuite lieu de remarquer que, même dans ces occasions réglées, toutes les paroles ne se valent pas : celle du roi, généralement ultime, appelle un assentiment systématique ( «koe!») et ne souffre aucune discussion. En contraste, toute parole qui lui est destinée doit être mise à distance, nécessite une médiation et doit humblement requérir un accord préalable.

Nous allons voir que les règles de l'échange formel jouent également lors de la cérémonie du kava. L'importance rituelle de l'occasion est telle, cependant, que les principes se renforcent et se complexifient donnant à saisir, mieux qu'en toute autre circonstance, la hiérarchie des statuts et des responsabilités au sein de la société wallisienne locale.

\section{Distribution du kava : manifestation de la hiérarchie des statuts et des responsabilités}

Pour importante qu'elle puisse être, la fonction de médiation assurée par le molofaha, lors des visites formelles peut être déléguée en l'absence de Nikola Vaitanoa. Elle n'apparaît pas non plus comme essentielle pour les Wallisiens qui, de façon très générale, définissent sa charge comme étant la distribution des coupes lors des cérémonies du kava royal. Il est intéressant, en effet, de voir à quel point, pour Nikola Vaitanoa comme pour les chefs coutumiers qui lui ont confié cette tâche, la gestion de la cérémonie du kava prime sur toutes ses autres responsabilités ${ }^{17}$. Cette importance apparaît nettement quand la dimension biographique et les questions statutaires sont abordées. Une analyse rapide des attributions du molofaha lors des cérémonies permettra de dégager les principes de sa fonction et le type de médiation qu'elle assure dans ce contexte précis.

L'officiant cérémoniel assurant la préparation et la distribution du kava royal porte normalement un nom-titre transmis dans une des trois parentèles dans lesquelles on hérite normalement de la charge. Le prédécesseur était Tino Tu'iha'atala dont le nom de fonction est devenu un patronyme. Le processus fut différent pour Nikola Vaitanoa. Quand il a repris la charge, la famille du prédécesseur s'est opposée à la transmission d'un titre (et d'un nom) qui lui appartenait. Bien que l'administration et une partie de la population continuent à le lui accorder, il y a renoncé pour faire du nom de sa fonction un nom dont on use dorénavant comme d'un titre (Molofaha, ou Nikola Molofaha). Le processus fut progressif et s'achève du fait de son long exercice dans cette charge. Aujourd'hui, certaines personnes le respectent et le craignent comme un membre de la chefferie, raconte-t-il amusé. Il y est toutefois étranger, formant, nous le verrons, le pendant cérémoniel de l'officiant (Tu'i'Uvea) chargé de la sécurité physique du roi et de l'exercice de la force lorsqu'il s'agit d'imposer une décision de la chefferie. 
Nikola Vaitanoa n'a toutefois pas hérité d'une charge ancestrale. Il fut choisi pour son investissement et ses compétences en matière de préparation du kava. De tels recours ne sont pas rares, le critère généalogique devant, dans l'idéal, se combiner avec les "compétences manifestes» ('aoga) d'un candidat. À défaut de trouver la bonne personne dans les parentèles détenant charge et titre, il arrive que l'on recoure au choix d'outsiders remarqués pour leurs qualités personnelles ${ }^{18}$. Jusqu'à un passé récent, rien de ce qui était pensé comme "traditionnel», à Wallis, ne passait par des apprentissages formels ${ }^{19}$. Les circonstances ont, dans ce cas précis, présidé à ce choix inattendu, Nikola Vaitanoa ayant remplacé avec succès l'officiant en charge un jour d'absence.

Après la mort de Tino, explique-t-il, on m'a demandé de descendre [sur l'esplanade du palais] pour faire un essai. Et je l'ai fait jusqu'à aujourd'hui !

Mais Sophie, continue-t-il plus loin, c'est comme en toute chose, tu en viens à t'habituer, tu t'habitues au travail et tu progresses toujours un peu, et tu réfléchis... J'ai commencé, Sophie, il y avait des aspects [de la cérémonie] qui m'ont donné du mal. Je n'avais pas encore bien compris la disposition de la chefferie : qui est devant, qui est derrière. Il y a eu des occasions, j'ai fait boire Kulitea puis, derrière lui, j'ai fait boire Fotu'atamai, après Kulitea! Kulitea en premier $^{20}$ ! Et puis j'ai compris... [...] qu'il y avait six coupes kava'aliki à l'avant du cercle, celle-ci la première, suivie de celle-là, suivie de celle-là, suivie de celle-là, suivie de celle-là. Je n'avais pas bien compris. Ce que j'avais retenu, c'était les paroles de l'ancien qui..., du distributeur, Tino. Mais je ne n'avais pas retenu qui était le premier membre de la chefferie à boire derrière le roi !

C'est donc progressivement que Nikola Vaitanoa a développé une expertise aujourd'hui incontestée. Un point mérite d'être souligné d'emblée, qui prendra toute son importance dans les développements qui suivent: il fut dépositaire de la charge avec l'accord et le soutien du Conseil royal, Kivalu, le "premier ministre", avalisant cette attribution sans la remettre en question suite à ses errements en matière de distribution. En d'autres circonstances, nous le verrons, il eut été contesté et probablement remplacé, mais ce soutien, malgré la répartition désordonnée des coupes parmi les membres du conseil, tend à montrer que l'essentiel de la tâche n'était pas là en ce moment de transition. Une revue point par point des attributions du molofaha lors du kava royal permettra de comprendre en quoi la distribution n'est qu'un aspect de responsabilités, dans les faits, plus étendues.

Il a été dit plus haut que le molofaha est indispensable à la cérémonie du kava royal. Assis aux pieds du roi, il en règle l'organisation et assure la juste répartition des coupes de boisson. Cette répartition organise, entre le premier (fuga kava) et le dernier service (hu'a kava) une alternance entre coupes aristocratiques (kava'aliki) et coupes valorisantes (kava matāpule ${ }^{21}$ ) : la première coupe est celle du roi, clairement distinguée par son nom, son annonce, et sa présentation. Suivent ensuite celles du Conseil, entre lesquelles s'intercalent celles (kava matāpule) des chefs de village. Suivent les coupes destinées aux officiels et aux invités assis autour du roi et de son Conseil, à l'avant de l'aire cérémonielle ('alofi), puis celles des anciens qui, assis de l'autre côté de l'aire cérémonielle, derrière le plat à kava lui faisant face, représentent le village qui offre le kava. Toutes ces coupes, y compris la dernière - la plus élevée en statut après celle du roi -, sont des coupes aristocratiques (kava'aliki)

36 Nikola Vaitanoa a tenu à m'expliquer pourquoi, une fois les règles suivies par son prédécesseur comprises et intégrées, il les a modifiées pour adopter cette nouvelle distribution : autrefois « tous les [kava]'aliki avaient leur [kava] matāpule ». Une longue 
explication suivit dont il ressort qu'il a mis fin à une alternance entre kava'aliki et kava matāpule qui s'étendait à toute la distribution, de part et d'autre de l'aire cérémonielle. Cette alternance générait la lassitude, car Tino, son prédécesseur, devait trouver de loin, dans l'assistance, les personnes susceptibles de recevoir les kava matāpule. Elle manquait, en outre de régularité, des coupes ne pouvant être attribuées dans le devant de l'aire cérémonielle étant ponctuellement attribuées à l'arrière. Autre problème, Tino continuait cette alternance parmi les représentants de l'État et les chefs de service présents sans considération pour leurs statuts relatifs. Enfin, elle était source de mécontentement en ce qu'il arrivait à Tino d'attribuer à des jeunes gens sans titre ou position des kava matāpule alors qu'ils pouvaient prétendre, par leur statut personnel, à des kava'aliki. Cette alternance généralisée favorisait une confusion entre statut personnel et position socialement validée, qui a conduit plusieurs récipiendaires à refuser une coupe en versant à terre le contenu. Dans ce désordre, il arrivait que certains participants boivent à deux reprises, contre toutes les règles

Je suis alors allé voir [...] le [représentant du roi] faipule Alemani pour en discuter et je lui ai dit qu'il fallait distribuer une seule sorte de coupes [suite à l'alternance respectée pour la seule chefferie]. Et je lui ai demandé si c'est une faute de finir [l'alternance] aux coupes des chefs de village et de distribuer toutes les autres coupes en suivant : « ho ku heka! ho ku heka... » [Annonces pour les coupes kava'aliki].

Cette simplification va à l'encontre de la séparation et de la complémentarité autrefois manifestée par l'alternance généralisée des coupes entre les "anciens" servis de kava'aliki et leurs "cadets", destinataires de kava matāpule; de nos jours, cette alternance est réservée à la seule chefferie, dans la suite immédiate de la coupe royale. Elle a, en revanche, introduit de la rigueur en ce que les erreurs sont évitées et les statuts personnels respectés. Elle s'accompagne d'une préparation minutieuse en amont de la cérémonie : Nikola Vaitanoa s'informe sur les présents, demande qui sont les «anciens » des différents groupes de parenté qui devront boire dans l'arrière de l'aire cérémonielle (tau'a), veille à ce que toutes les places soient correctement occupées et surtout invite chacun à prendre la place qui correspond à son statut et, par conséquent, à la coupe qui lui sera attribuée lors de la distribution.

Le distributeur doit savoir si quelqu'un est mal placé. Par exemple s'il [le récipiendaire d'une coupe kava'aliki] vient s'installer sur le rang de devant [dans lequel on distribue des kava matāpule], il est mal placé! [...] Alors ce que je fais toujours auprès des chefs de village, des chefs de district, je mets de l'ordre à l'arrière de l'aire cérémonielle, pour faire venir devant ceux qui ne sont pas 'aliki. C'est une source d'erreur! Parce que si j'attribue [à un 'aliki] une coupe qui ne lui est pas due, c'est comme si je le rabaissais et il la renverse devant lui !

Dans un contexte où la cérémonie met en représentation, spatiale et temporelle, les statuts socialement validés par des fonctions et/ou des responsabilités, ces préparatifs préviennent les erreurs ou les ambiguïtés. Contrairement à ce qui se passait du temps de Tino, positions dans l'espace et ordre des coupes dans la distribution doivent correspondre. Dans les rares cas où quelqu'un est assez déterminé ou soutenu pour braver cette règle, le molofaha a la responsabilité de trouver une solution. Une première solution est de ne pas attribuer de coupe, infligeant un affront cinglant au contrevenant :

Mais si un homme prend une mauvaise place, une place qui ne lui convient pas, qu'il s'assied là alors que sa place est ailleurs, si je lui dis de partir et qu'il ne le fait pas. Cette question revient à la distribution du kava. Je peux alors ne pas le faire boire, même si c'est un chef ['aliki]. Cela dépend de moi. Pour ce qui est de faire 
boire les chefs ['aliki], s'il est juste que je n'en fasse pas boire un, je ne le fais pas boire. C'est moi qui décide en la matière.

tant que ses décisions sont motivées et justifiables. Dans cette limite, qui implique un service juste et attentif, sa charge le distingue et l'ennoblit aux yeux de la population et de la chefferie. Il en va ainsi de toute charge coutumière. Ici, l'évaluation des compétences du molofaha et son appréciation rejoignent celles valables pour tous les porteurs de titre. En l'occurrence, la confiance et le soutien de la chefferie ont fini par conférer à son exécutant une autorité considérable :

Les responsabilités qui m'incombent, je suis seul à les prendre, moi seul. Quand arrive le moment du kava personne d'autre ne décide quant à la cérémonie. Y compris le roi ! Il n'est plus possible à quelqu'un de me donner un avis sur les tâches qui m'incombent, je suis seul à diriger le kava. L'ensemble de la chefferie [n'a rien à dire]. S'il est juste que je ne fasse pas boire un de ses membres, il ne boit pas! Mais au cours de ma carrière, Sophie, plus de vingt ans, je n'ai jamais... Je distribue à la chefferie, ils n'en furent jamais mécontents.

Ainsi, lorsque le roi Tomasi Kulimoetoke mourut après cinquante ans de règne, il eut carte blanche pour organiser les cérémonies du kava funéraire et de l'investiture de son successeur, Kapeliele Faupala, dont on ne connaissait plus le détail du déroulement.

Le molofaha prend donc un soin particulier à l'organisation de cérémonies de façon à ce qu'elles se passent sans incident. Car, explique-t-il, «On sait, selon le déroulement du kava, si le pays vit bien, ou s'il vit mal. S'il y a des troubles. » Dans les cas où des événements échapperaient à son contrôle, il est probable qu'ils renverraient à des troubles graves dont le règlement échapperait à la gestion maîtrisée des positions et des statuts ou de la distribution des coupes. 


\section{Organisation des cérémonies et obtention des bienfaits divins}

En matière de kava et de cérémonies, la fonction du molofaha ne s'arrête pas à la distribution de coupes, certes l'aspect le plus technique de son office. Il est, en effet, responsable de l'ensemble du rituel, du début jusqu'à sa fin.

C'est moi qui annonce le moment de commencer la cérémonie. Alors, je préviens Pulu'i'Uvea [officiant gardien de l'aire cérémonielle]. Pulu'i'Uvea descend vers le fond de l'aire cérémonielle, s'assurant que tout est en place [tout le monde doit être assis à terre, le silence doit régner] puis on fait la cérémonie. Il revient à Pulu'i'Uvea de dire aux gardiens qu'ils aillent faire s'installer la foule. Si je ne vois pas Pulu'i'Uvea, je le dis directement.

Car

[...] lorsque je suis devant [à diriger la cérémonie], la charge de Pulu'i'Uvea est [à l'opposé de l'aire cérémonielle,] de garder l'avant à partir de l'arrière. Il garde l'aire cérémonielle, le rassemblement, c'est lui qui est garant du bon ordre. S'il voit quelque chose [qui ne va pas], il l'empêche. Mais il ne partage pas mes responsabilités.

Le rôle du molofaha s'étend donc en amont et en aval de la distribution du kava : Le pouvoir du distributeur..., je parle de l'essentiel de ma charge, le pouvoir qui m'est conféré, je décide de ce qu'il faut faire et ne pas faire. Par exemple, le temps du partage des offrandes de vivres, cela dépend de l'avant, de mon autorisation pour engager cette phase. Pour le temps des danses, il dépend de moi d'autoriser les danses.

C'est à son appel que le chef de district s'approche du roi pour présenter la composition de l'offrande et annoncer son partage. Ici, la fonction du molofaha revient à la médiation étudiée dans le cadre des visites formelles faites au roi. À son invite, le chef du district quitte sa place initiale, à l'opposé de celle du roi, et vient s'asseoir juste devant le molofaha. Il use de la même formule que lors des visites, pour obtenir son autorisation d'opérer la distribution: "Molofaha s'il te plaît, voici les humbles enfants de Lavelua qui accourent et désirent te demander la permission, si tu le veux bien... » Il en va de même, ensuite, pour les danses. Organisateur de l'espace et du temps de la cérémonie, garant d'une juste distribution des coupes de kava, le molofaha retrouve ici sa fonction régulatrice et médiatrice, bien qu'il ne s'agisse plus d'une adresse au roi, mais de la présentation et de la distribution des offrandes du jour.

Pourtant, une fois encore, ses attributions vont plus loin. Il assure, au cœur de la cérémonie, les conditions de la prospérité générale en ce qu'il contrôle les discours et assure les prières afin que Dieu bénisse à travers le roi, l'ensemble du pays formé par l'assistance réunie, en retour des offrandes du jour.

Lors de la cérémonie elle-même, la préparation du kava est ponctuée par deux importants discours. L'un d'eux, appelé pulou te fa'u, est prononcé par un membre de la chefferie, parfois un prêtre, en référence à l'occasion de la cérémonie. Mais, explique Nikola Vaitanoa,

il faut que je sache qui va parler, au moment du pulou te fa'u. Avant le kava, je demande qui parle, qui fait le discours. Puis au moment du pulou te fa'u, je le préviens : «c'est maintenant!». Et au moment où je fais s'avancer Kivalu [s'il lui revient de parler], je lui dis «c'est ton moment !» jour de la fête de territoire, ce fut un simple réglage. Le Conseiller pressenti pour le 
discours n'étant pas francophone, le molofaha demanda, par courtoisie envers le Préfet, qu'un autre Conseiller prenne sa place.

Car il dépend de moi, de ma responsabilité à l'avant, c'est comme cela que nous le concevons. J'écoute les paroles de l'homme. Il agit d'une façon que je trouve correcte et qui correspond à l'occasion, je regarde comment il travaille, sa façon de... et si je vois qu'il est bien, je... le laisse faire le discours du kava.

Selon les circonstances, son intervention peut donc être plus lourde : s'il pressent - ou sait - que l'orateur désigné ne sera pas bienveillant envers le roi, qu'il peut engendrer des troubles ou commettre un affront, il peut le remplacer en appelant un autre orateur. Dans un cas extrême, il lui est arrivé de suggérer au souverain qui lui demandait son avis la destitution d'un Conseiller qui, en état d'ébriété semble-t-il, n'avait pas su se tenir lors de la cérémonie et avait prononcé un long discours parfaitement hors de propos.

51 L'autre discours prononcé lors du kava prend la forme d'une prière. Il est appelé l'offrande du kava (momoli o te kava). Il se compose de formules que le molofaha prononce lentement et de façon réglée sur les gestes rythmés du préparateur lorsqu'il manipule le filtre de fibres destiné à purifier la boisson de ses impuretés.

Le moment où l'on fait l'offrande du kava, [lors de] son filtrage et tout cela, je ne parle pas au nom de... je le fais pour la chefferie, je demande à Dieu pour le roi et son Conseil. Car quand je fais la demande, je ne la fais pas en leur nom, je la fais comme un homme en prière, comme un prêtre. Je la fais pour toute l'assemblée présente et la chefferie.

Il reprend plus loin :

C'est comme si j'étais un prêtre... qui dit la messe pour tout le monde... pour intercéder en la faveur de tous. Ainsi, au moment de l'offrande du kava, c'est moi qui fais la prière pour la prospérité de tous, que le roi se porte bien ainsi que son Conseil, ainsi que tout le pays. Car c'est une chose qui nous vient d'autrefois! Qui vient des temps païens. Car l'homme qui présente les offrandes, il les offre comme on les offrait à Tagaloa ${ }^{22}$, à... C'est ainsi ! On présentait les offrandes à Tagaloa afin qu'il favorise les jardins, toutes les choses...

52 Cette prière reprend donc une pratique préchrétienne, autrefois destinée à Tagaloa, déité prééminente parmi les entités qui présidaient aux destinées des chefs et de leurs dépendants. De nos jours, le kava est présenté à Dieu, la prière de l'offrande demandant :

Laissez-nous profiter des bienfaits de la mer, ainsi que de la végétation... de décisions judicieuses, de la prospérité apportée par le travail, du bon gouvernement du pays...!

Ainsi, je fais l'offrande du kava et des présents, que Dieu les bénisse, que le roi y prenne sa coupe, c'est béni vraiment, on dit que Dieu les a bénis.

Le kava offert à Dieu est bu par le roi, car

c'est son représentant ! [...] Lors des cérémonies traditionnelles, la cérémonie du kava est une cérémonie traditionnelle ! On fait l'offrande traditionnelle, il revient à un homme tel que moi, qui suis le maître du kava, de l'offrir en mon nom, car c'est à moi qu'il revient de le faire boire, de l'offrir et d'obtenir une bénédiction sur tout ce dont il retourne ici. Pour cette raison je parle directement à Dieu pour le prier de protéger et de bénir le kava, qu'il assure la prospérité du roi et de son Conseil, ainsi que de tout le peuple du pays.

Au-delà donc de l'organisation générale de la cérémonie (organisation de la succession des coupes, gestion des inadéquations et des inaptitudes, validation des discours), il 
revient donc au molofaha d'assurer, par sa prière, la prospérité générale, confortant par là l'assise rituelle et la légitimité du roi.

Si nous revenons maintenant au contraste et à la conjonction des fonctions de molofaha et de mu'a, nous voyons mieux en quoi elles se rejoignent par l'agencement des relations qu'elles rendent manifestes.

55 La fonction du molofaha est double : i) il maîtrise la parole et assure la médiation rituelle lors des échanges formels et ii) il organise la cérémonie du kava royal lors des grandes occasions. Cependant, si N. Vaitanoa peut être ponctuellement remplacé auprès du roi dans son rôle de médiateur, il ne peut l'être sans risque de graves désordres dans l'organisation d'une cérémonie du kava royal. Cette fonction n'est pas sans ressembler à celle des prêtres qui, en Polynésie orientale, veillaient au culte des déités et organisaient les temps forts du calendrier rituel. Pour l'essentiel, il semble bien que la fonction de molofaha réside, à Wallis, dans la distribution des coupes et dans les responsabilités rituelles, notamment dans la gestion des discours - y compris de la prière - que cela comprend.

56 La fonction de $m u$ 'a consiste en un accompagnement, le mu'a allant devant, s'assurant moins de la sécurité que du respect des règles relatives aux interactions avec le roi : respect des distances et des règles d'évitement très strictes relatives au roi, contrôle de la qualité des présents offerts. Cette qualité n'est pas tant intrinsèque aux présents, bien que seul le meilleur des offrandes soit réservé au roi. Elle réside dans la médiation elle-même. Ainsi, lors de la cérémonie du kava, la première coupe (fuga kava), celle réservée au roi, est toujours distinguée selon deux modalités complémentaires : cette coupe, appelée taumafa, est signalée, lors de son attribution, par une formule particulière « kava kā heka » et non " heka » ou " hā ku heka » comme c'est le cas pour les autres coupes. Son autre particularité, qui me fut rappelée plusieurs fois par l'entourage de Nikola Vaitonoa lors de l'entretien, est de ne pas être directement servie au roi : présentée à deux mains (à la différence de toutes les autres), elle est remise à l'officiant assurant la distribution (molofaha). Celui-ci en verse le contenu dans la coupe personnelle du roi vers qui il se tourne pour la lui remettre dans un second temps. Ce service en deux coupes et en deux temps, unique dans la cérémonie, correspond à la façon dont la parole doit être transmise au roi pour être audible et valide.

57 Dans les deux cas l'interposition consiste à servir et à protéger, non pas tant la personne royale (comme le feraient un orateur délégué dans le premier cas, des éclaireurs ou des goûteurs dans le second) que la dignité et l'autorité de la fonction royale, à l'intersection de la société des vivants et du monde des ancêtres, dominé par le Dieu chrétien, dont dépend la prospérité générale. Dans le cas du mu'a, cependant, l'accent semble mis sur la gestion des interdits relatifs à la personne du roi et à la préservation de sa fonction. Sa médiation distingue formellement le roi de ses dépendants, signifiant la distinction en valeur qui est reconnue à celui dont dépend la prospérité de tous en vertu de sa position intermédiaire et des responsabilités rituelles qui lui incombent. Dans le cas du molofaha, tout au moins lorsqu'on le considère dans sa fonction principale de distributeur de kava, la médiation semble tournée vers l'au-delà, sous la présidence du roi, alors résolument situé parmi les vivants, face aux ancêtres et à Dieu, convoqués pour l'occasion. Il semble qu'avant la christianisation, un prêtre ou un descendant de l'ancêtre convoqué pouvait prendre la présidence de la cérémonie, à l'interface des deux domaines, c'est-à-dire, à la place du roi. Le molofaha fait alors office d'officiant rituel face à l'au-delà que représente le roi parmi les vivants (dans une 
moindre mesure sa chefferie et désormais les prêtres catholiques). C'est de toute l'assemblée qu'il fait monter la prière (roi, prêtres, chefferie compris) demandant, en retour, les conditions de la prospérité générale et du juste retour des bienfaits obtenus, autorisant leur répartition secondaire sous forme de kava, de vivres, de divertissements ${ }^{23}$.

\section{Le contrôle des relations et l'expression de l'asymétrie} visites auprès du roi, montrent une forme particulière d'adresse indirecte aux entités pensées comme supérieures. Il s'agit là, couplée aux règles d'évitement et à l'usage du niveau de langue approprié, d'une forme originale d'institutionnalisation de la parole indirecte : cette forme d'adresse repose sur une médiation qui assure, avec le contrôle des relations mises en présence et ainsi exprimées, le respect de leur asymétrie fondamentale. hiérarchisés en valeur: les Wallisiens partagent avec d'autres sociétés polynésiennes l'idée que le destin des déités et des morts dépend du culte qui leur est porté, le monde des ancêtres et l'ensemble du cosmos trouvant leur origine dans la société et dans la vie qui anime groupes sociaux et relations. Cependant, de façon très immédiate, les hommes et leur destin en ce monde dépendent du bon vouloir des ancêtres, des déités reconnues et, depuis plus d'un siècle, du Dieu chrétien introduit dans la vie locale par les missionnaires catholiques. C'est pour obtenir leur soutien et la fertilité qui en découle que divers rites étaient autrefois et sont encore, dans une certaine mesure, pratiqués. La cérémonie du kava doit être comprise comme un temps fort de cette mise en relation des deux domaines. Une prière (te momoli o te kava) est portée vers l'au-delà, sous la présidence et la responsabilité du roi qui, garantissant la hiérarchie des hommes et des groupes adossée à son statut apical (hiérarchie explicitement exprimée lors de la distribution des coupes), rend sensible le bon ordre de la société tandis que les distributions de vivres et d'argent manifestent la prospérité du "pays» que constituent ensemble vivants et morts.

Dans cette configuration, le roi est en position d'ancien absolu, plus proche que tout autre des ancêtres et de Dieu. Cette position doit être à la fois signifiée et protégée des atteintes, en actes et en parole (violence et défi des interdits) par des formes de médiation qui préviennent un accès direct : offrandes et parole ne doivent lui parvenir directement, une médiation étant nécessaire. Une telle médiation signale la différence en valeur dans les pôles de la relation mobilisée. Le roi, par ses compétences, sa droiture, sa foi, obtient les bienfaits provenant de l'Au-delà au bénéfice de tous. Dans ce sens, il constitue le relais entre les ancêtres dont il forme une actualisation parmi les vivants et son peuple. Ce dernier, par son intermédiaire, reçoit ces bienfaits, immédiatement visibles dans la prospérité générale et le bon ordre du "pays ». Dans l'autre sens, l'asymétrie est plus nettement signifiée. Pour ses dépendants, le roi appartient au pôle supérieur, en statut comme en valeur. La relation qu'il établit avec l'Au-delà n'est équivalente à aucune autre dans la société wallisienne : une médiation rituelle est alors nécessaire qui, signifiant la distance, l'instaure et conforte la fonction royale dans sa propre situation intermédiaire. En l'établissant, elle la préserve et en protège le caractère particulier, « séparé » (tapu). 
61 Remarquons, pour conclure, que cette médiation rituelle suit des règles simples (modalités rigides et formules préétablies) qui autorisent à l'officiant cérémoniel le jeu d'adaptations périphériques aux contextes et aux situations. On en a vu quelques exemples ponctuels avec les corrections qu'officiant et participants tentent d'apporter, lors du kava, au positionnement de chacun dans l'espace, mais aussi, avec la transformation plus durable des séquences dans la distribution du kava ou encore le changement de préséance au sein de la chefferie. Ici, comme ailleurs dans le monde social wallisien, le rite n'est pas rigide. Il laisse une marge à l'expression des acteurs, au jeu de leurs interactions, et aux mutations de l'ordre et des valeurs sociales qui en résultent.

\section{BIBLIOGRAPHIE}

BATAILlon, Pierre, 1841, Notice sur la mission de Wallis, Annales de la Propagation de la Foi 13, Lyon, p. 5-24.

BLIXEN, olaf, 1966, El language honorifico en Uvea (Wallis) y sus connectionnes en Polynesia occidental, Moana, Estudios de Anthropologia Oceanica, $\mathrm{n}^{\circ}$ 1, Montevideo, p. 1-15.

BRENNEIS, DONALD L. \& Myers, FRED, 1989, Dangerous words: Language and Politics in the Pacific, New York and London, New York University Press.

BURRows, Edwin Grant, 1937, Ethnology of Uvea (Wallis Island), Honolulu, Hawai'i, Bernice P. Bishop Museum Bulletin, no 145.

CHAVE-DARTOEN, Sophie, 1992, La cérémonie du kava à Wallis (Polynésie occidentale), Mémoire de DEA en Anthropologie sociale, Paris, EHESS.

CHAVE-DARTOEN, Sophie, 1997, Étude de la natte ta'ovala à Wallis (Polynésie occidentale), in Y. Broutin (éd.), Cahiers de Linguistique sociale, «Se vêtir pour dire », coll. « Bilans et Perspectives ", p. 61-75.

CHAVE-DARTOEN, Sophie, 2000, Uvea (Wallis), une société de Polynésie occidentale, étude et comparaison, Thèse de doctorat en Ethnologie et Anthropologie sociale, Paris, EHESS.

CHAVE-DARTOEN, Sophie, (à paraître), Circulations monétaires à Wallis (Polynésie occidentale), communication au séminaire "Monnaies et identité », Université François Rabelais de Tours (CeRMAHVA, IRAMAT), 26 mars 2011.

CHAVE-DARTOEN, Sophie, (à paraître b), Le sang et le travail. Aux sources de l'autorité dans le royaume de Wallis, in $\mathrm{D}$. Gibaut et $\mathrm{S}$. Vibert, ouvrage collectif en hommage à $\mathrm{D}$. de Coppet (titre non défini).

Duranti, ALESSANDRO, 1988, Intentions, language, and social action in a samoan context, Journal of pragmatics 12 , pp. 13-33.

Duranti, ALESSANDRO, 1990, Doing things with words: conflict, understanding and Change in a Samoan fono, in Karen Ann Watson-Gegeo et Geoffrey M. White (eds), Disentangling. Conflict discourse in Pacific Societies, Stanford California, Stanford University Press. 
Duranti, ALESSANDRO, 1994, From Grammar to Politics: Linguistic Anthropology in a Western Samoan Village, Berkeley and Los Angeles, University of California Press.

FIRTH, Raymond, 1970, Postures and Gestures of Respect, in J. Pouillon et P. Maranda (éds), Échanges et communication. Mélanges offerts à Claude Lévi-Strauss, La Haye, Mouton, p. 188-209. GOFFMAN, Erving, 1991, Les cadres de l'expérience, Paris, Éditions de Minuit.

GIFFORD, Edward W., 1929, Tongan Society, Honolulu, B. P. Bishop Museum Bulletin, no 61 [reed., 1985].

GUNSON, Niel, 1979, The Hau Concept of Leadership in Western Polynesia, Journal of Pacific History, vol. 14 , pp. 28-42.

HENQUEL, Père Joseph, Circa 1910, Talanoa ki Uvea nei, manuscrit dactylographié et ronéotypé, Wallis, Évêché de Lano.

HOCART, Arthur M., 1978, Roi et courtisans, Paris, Le Seuil.

MARCUS, George E., 1980, Role Distance in the Conversations Between Tongan Nobles and their 'People', Journal of the Polynesian Society, vol. 89, pp. 435-453.

MARCUS, George E., 1989, Chieftainship, in A. Howard \& R. Borofsky (eds), Developments in Polynesian Ethnology, Honolulu, University of Hawai'i Press, pp. 175-209.

MARTIN, John D., 1981, Tonga Islands, William Mariner Account, 4th ed., Tonga, Vava'u Press [1st ed., London, 1817].

MONNERIE, Denis, 2005, La parole de notre maison. Discours et cérémonie kanak aujourd'hui, Paris, CNRS Éditions, Éditions de la Maison des Sciences de l'Homme.

O'REILLY, Patrick, 1948, La religion des Polynésiens, Histoire générale des religions, t. I, Paris, Aristide Quillet, p. 139-155.

SAHLINS, Marshall, 1995, How the "natives" think about Captain Cook for example, Chicago, The University of Chicago Press.

TCHERKEZOFF, Serge, 2003, Fa'a-Samoa. Une identité polynésienne (économie, politique, sexualité).

L'anthropologie comme dialogue culturel, Paris, L'Harmattan.

TURNER, Victor, 1974, Dramas, Fields, and Metaphors, Ithaca, Cornell University Press.

YANAH, Kwesi, 1995, Speaking for the chief: Okyeame and the politics of Akan royal oratory, Bloomington \& Indianapolis, Indiana University Press.

\section{NOTES}

1. Ce travail n'aurait pu être mené sans la bienveillante participation de Nikola Vaitanoa, assistant cérémoniel du roi de Wallis, de son épouse, Laime, de leurs proches Leone Vaitanoa et Makalita Bringold qui ont pris une part active à l'enquête. Ma reconnaissance aux enfants, pour leur présence attentive lors des longues discussions. Merci à Elisio Dartoen qui m'a aidée pour affiner certaines traductions.

2. Par leurs fonctions et leurs attributs, des orateurs samoans sont comparables, à plus d'un titre, aux orateurs akans (Yankah, 1995) : ce sont des médiateurs «agissant par la parole à la place» du roi (surrogate performance), qui maîtrisent les émotions et les techniques de communication. Mentionnons que ces derniers maintiennent, en outre, les visiteurs à distance du 
dangereux contact de la personne royale, ce qui n'est pas exactement le cas à Wallis, comme nous le verrons plus loin dans le texte.

3. Synthèse à partir de Gifford (1929) et Martin (1981). De tels officiants existent encore à Tonga, mais leurs responsabilités cérémonielles et rituelles se sont modifiées depuis l'instauration, au $\mathrm{XIX}^{\mathrm{e}}$ siècle, d'une monarchie constitutionnelle inspirée du modèle britannique.

4. Sous le même terme de hau différentes formes d'autorité sont désignées selon les sociétés polynésiennes considérées (Gunson, 1979). Dans le proche archipel de Tonga, le hau est glosé comme le chef exécutif, bras armé du "roi sacré» tu'i. On ne retrouve pas si nettement distinguées les deux fonctions à Wallis où la chefferie actuelle, bien que d'origine tongienne, connut ses propres développements (Chave-Dartoen, à paraître (b)). Les termes français de "royauté » ou de "roi », sont impropres à une traduction satisfaisante, mais couramment employés par les Wallisiens eux-mêmes en référence à ce qui est en fait un "chef suprême ». Je me plierai à cet usage sans plus user de guillemets dans la suite du texte.

Le roi (hau) wallisien constitue l'apex d'une chefferie constituée d'un Conseil de six membres ('aliki fa'u : Kalae Kivalu, Mahe Fotu'āika, 'Uluimonua, Kulitea, Fotu'atamai et Muko'ifenua...), de vingt et un chefs de village (pulekolo) et de trois représentants (faipule) relayant son autorité dans les districts. Lors de la cérémonie du kava, chacun d'eux a une place et une coupe dont l'ordre de distribution suit la hiérarchie des statuts.

5. À la préséance dans les tours de parole correspond le droit au dernier mot. La parole de la personne ayant le statut le plus élevé est terminale si elle n'invite pas à une réponse (cf. infra dans le texte). Le débat contradictoire n'a que très peu de place dans les discussions formelles. En cas de litige par exemple, comme lors des délibérations du conseil, chacun est invité à exposer son point de vue, puis la question est tranchée par le chef, ici en position d'arbitre, sans que l'on puisse y revenir.

6. tapu est généralement traduit par « sacré » ou « interdit ».

7. Nikola Vaitanoa indique que la pratique change, et que les membres du Conseil lui demandent parfois de l'accompagner...

8. Martin $(1981,353)$ insiste sur l'importance de la face, à Tonga, comme manifestation de la présence. Tourner le dos marque une absence. Il est significatif qu'en contexte formel, les chefs présentent ainsi deux faces : la leur propre, qui est doublée par l'assistant cérémoniel (matāpule «face-de-chef ", mu'a « devant ») installé devant lui.

9. Cette idée est développée dans les traditions orales compilées au début du siècle par le père Henquel en un recueil qui circule dans l'île et qui est étudié à l'école.

10. À Wallis, le terme signifie "avant», "devant», mais désigne plus précisément dans ce contexte l'« aide d'un roi, le bras droit d'un roi ». Son sens est proche à Tonga, où il désigne en outre un ordre social intermédiaire que constituaient les assistants cérémoniels de second ordre des nombreux chefs de l'archipel... Une partie de notre discussion avec N. Vaitanoa a porté sur le lien historique entre Tonga et Wallis et sur les dissemblances entre les institutions des deux archipels.

11. Puipui signifie par extension "protéger, réserver l'accès ». Je n'ai jamais assisté à ce type de rencontre, description et analyse s'appuient ici sur le récit de N. Vaitanoa et des proches présents lors de l'entretien. L'analyse de l'espace constitué lors de toute occasion formelle montre qu'il est conçu comme fermé, les participants se faisant face. Dans ce contexte, le «chef » présente, dans la configuration formée avec son officiant (molofaha, mu'a), une double face, une présence en quelque sorte redoublée : celle immédiatement accessible (l'officiant), et celle, plus distante et impénétrable, du «chef » que l'on n'approche pas et dont on évite de croiser le regard (ChaveDartoen, 1992, 2000 ; Tcherkezoff, 2003).

12. Un secrétaire " chargé de communication » le remplace souvent auprès du souverain actuel.

13. Duranti (1988) a décrit ce type de phénomène pour Samoa et l'a analysé en terme de relations «politiques" selon deux focales différentes : les conseils samoans forment des social 
drama (Turner, 1974) où des groupes antagonistes cherchent à contrôler leurs actions politiques respectives (p.16). À une autre échelle, celle des acteurs sociaux pris dans leurs positions personnelles respectives, seule l'élévation du statut autorise l'expression de l'autonomie du sujet parlant. L'usage du sujet collectif permet la prudence oratoire (p. 25-26). Elle signifie de fait la reconnaissance d'une asymétrie en statut et en pouvoir.

14. Comme dans les sociétés proches, notamment à Tonga, un lexique particulier (quelques dizaines de mots) et des formules appropriées sont employés en adresse et en référence au roi (Burrows, 1937, 74; Blixen, 1966). À Wallis, ce registre a été emprunté par les missionnaires catholiques en référence et en adresse à Dieu, Jésus-Christ et la Vierge Marie.

15. Des informateurs issus des deux partis m'ont ainsi expliqué que, lors des événements de 2005 , il fut impossible de destituer le roi en place, car son entourage prévint toute tentative en interdisant aux opposants l'accès à sa personne. L'entrevue qui aurait permis de signifier une destitution selon les formes requises ne put avoir lieu.

16. En toute occasion, les Wallisiens sont anxieux de connaître le nom des inconnus afin de pouvoir les saluer correctement et de leur donner ainsi, avec une marque de respect, l'occasion d'exister pleinement dans les échanges sociaux. À défaut de savoir le nom, on use de formules convenues manifestant respect et intérêt en guise d'excuses lors des salutations.

17. Cela est également vrai à Tonga, alors qu'à Samoa, l'accent est mis, concernant ces officiants cérémoniels, sur leur fonction d'orateurs. Ce clivage a des racines culturelles, les chefferies locales s'étant développées selon des principes un peu différents, et historiques. Wallis fut conquis, puis resta longtemps dans l'orbite tongienne.

18. Cette règle est valable en principe pour l'accès à toutes les charges. Dans les faits, elle peut générer de vives tensions et donner lieu, pour ce qui est de la charge suprême (et du titre royal), à ce qui apparaît comme une usurpation.

19. Seules les chorégraphies de certaines danses et du brassage du kava font l'objet d'un apprentissage organisé. Mais dans les deux cas, les principes premiers sont considérés comme acquis et ne sont enseignés que les enchaînements.

20. L'ordre de distribution des coupes est normalement Kalae Kivalu, Mahe, 'Uluimonua, Kulitea, Fotu'atamai et Muko'ifenua.

21. Cette répartition peut être comprise comme étant, en posant une origine tongienne, celle intercalant la boisson des mu'a (appelé également matāpule à Tonga) respectifs entre les coupes destinées au chef ('aliki) afin, ici encore, de préserver la distance existant entre eux, et par là, leur caractère « séparé, préservé ». Elle implique surtout une alternance entre deux pôles constitutifs des relations d'autorité : les « anciens » qui, assurent les conditions de la prospérité générale en relation avec l'au-delà et leurs «cadets » qui valident leur position en leur assurant un soutien actif.

22. Tagaloa était, avant l'arrivée du christianisme, la déité première qui, ayant tiré l'archipel du fond de la mer, résidait au Ciel (Lagi). Tagaloa avait pour caractéristique de ne jamais avoir connu de vie terrestre, à la différence des nombreuses autres déités tutélaires.

23. Lors des danses, de très grosses sommes d'argent sont distribuées et réparties entre les danseurs, puis parmi leurs proches avant d'irriguer l'ensemble du réseau social. Il apparaît ainsi que les danses assurent, tout comme la cérémonie du kava et la «distribution des vivres» (katoaga), une forme - relativement récente - de distribution réglée et socialement validée (Chave-Dartoen, à paraître a). 


\section{RÉSUMÉS}

À Wallis (Polynésie), la supériorité de statut et l'asymétrie des relations qu'elle constitue impliquent une mise à distance spatiale, linguistique et rituelle qui suit différentes modalités en fonction des occasions formelles. L'une d'elles est la médiation spécifique qu'opère l'assistant cérémoniel appelé molofaha, particulièrement nécessaire dans toute adresse au roi. Cette étude présente différentes formes de la médiation rituelle qui tout à la fois protègent l'homme détenant la position royale, maîtrisent la parole qui lui est adressée et rendent manifeste l'asymétrie des relations existant entre ce roi, dispensateur des bienfaits que Dieu lui accorde, et l'ensemble de ses dépendants, bénéficiaires de sa bénédiction et des bienfaits ainsi obtenus.

In Wallis Island (Western Polynesia), status superiority constitutes a relationship asymmetry that implies spatial, linguistic and ritual modalities of distancing from the speaker depending on formal occasions. The specific mediation of the ceremonial attendant called molofaha is one of these modalities, when an address to the king is made necessary. This paper presents different forms of ritual mediation that preserve the man in kingly position, control the speech directed to him and show the asymmetry of the relations between this king, who dispenses the benefits granted by God, and the body of his followers, that rely on his benediction and the benefits then gotten on their behalf.

\section{INDEX}

Thèmes : anthropologie (Océanie)

Mots-clés : médiation rituelle, assistant (officiant) cérémoniel, asymétrie des relations

Index géographique : Wallis, Polynésie

Keywords : Ritual Mediation, Ceremonial Mediation, Asymmetric Relations, Wallis, Polynesia, Anthropology 